\title{
Chimeric ALT Plus TFL Perforator Flap for Breast Reconstruction Post Radical Mastectomy with Large Skin Defect
}

\author{
Dushyant Jaiswal ${ }^{1} \quad$ Mayur Raman Mantri ${ }^{1} \quad$ Vinay Kant Shankhdhar ${ }^{1, \odot} \quad$ Snehjeet Hemant Wagh ${ }^{1}$
}

${ }^{1}$ Department of Plastic and Reconstructive Surgery, Tata Memorial Centre, Homi Bhabha National Institute, Mumbai, Maharashtra, India
Address for correspondence Snehjeet Hemant Wagh, MCh (Plastic Surgery), Department of Plastic and Reconstructive Surgery, Tata Memorial Centre, Homi Bhabha National Institute, Mumbai, Maharashtra, India (e-mail:waghsh@gmail.com).

\begin{abstract}
Keywords

- anterolateral thigh flap

- tensor fascia lata flap

- post mastectomy reconstruction
\end{abstract}

\section{Introduction}

Breast cancer is the most common cancer in Indian women. ${ }^{1}$ Malignant phyllodes is a lesser common subtype. Radical surgery followed by radiation is the primary modality of treatment. With larger tumors, excision results in defects not amenable to primary closure or any oncoplastic maneuvers, needing skin replacement with a flap which is robust to tolerate radiation. Skin cover often becomes an endpoint of reconstruction rather than restoring breast mound. Conventionally, latissimus dorsi (LD) flap, anterolateral thigh (ALT) flap, and deep inferior epigastric artery perforator (DIEP) flap are used for resurfacing large mastectomy defects.
We present experience with chimeric free ALT flap plus tensor fascia lata (TFL) perforator flap for wound coverage and unilateral whole breast reconstruction.

\section{Case Report}

A 17-year-old girl presented with lump around $18 \mathrm{~cm} \times$ $11 \mathrm{~cm}$ occupying the entire left breast. Biopsy revealed malignant phyllodes tumor ( $\boldsymbol{- F i g}$. 1). She had undergone excision of left breast lumps thrice in the past 4 years with diagnosis of juvenile/cellular fibroadenoma. A radical mastectomy was performed with excision of overlying skin and underlying pectoralis major muscle, with margins of $2 \mathrm{~cm}$ published online June 28, 2021
DOI https://doi.org/

$10.1055 / \mathrm{s}-0041-1730844$ ISSN 0970-0358
(C) 2021. Association of Plastic Surgeons of India.

This is an open access article published by Thieme under the terms of the Creative Commons Attribution-NonDerivative-NonCommercial-License, permitting copying and reproduction so long as the original work is given appropriate credit. Contents may not be used for commercial purposes, or adapted, remixed, transformed or built upon. (https://creativecommons.org/licenses/by-nc-nd/4.0/).

Thieme Medical and Scientific Publishers Pvt. Ltd. A-12, 2nd Floor, Sector 2, Noida-201301 UP, India 

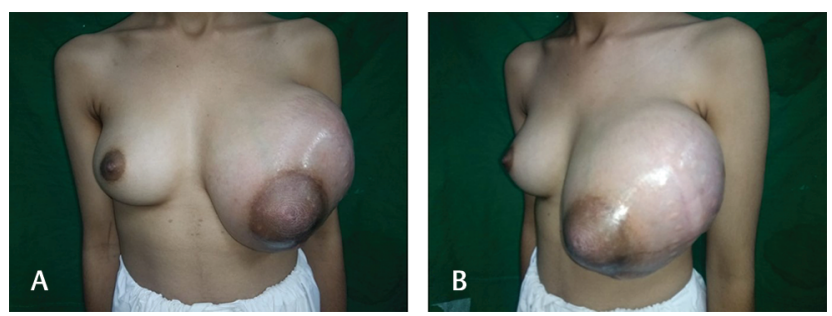

Fig. 1 (a,b) Preoperative photograph showing left breast phyllodes tumor.
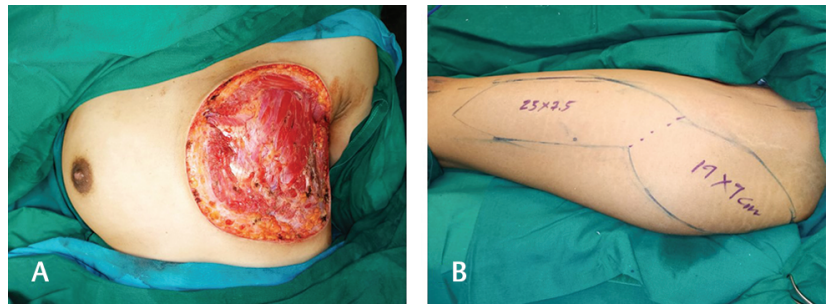

Fig. 2 (a) Mastectomy defect of size $20 \mathrm{~cm} \times 18 \mathrm{~cm}$. (b) Planning of ALT plus TFL flap.
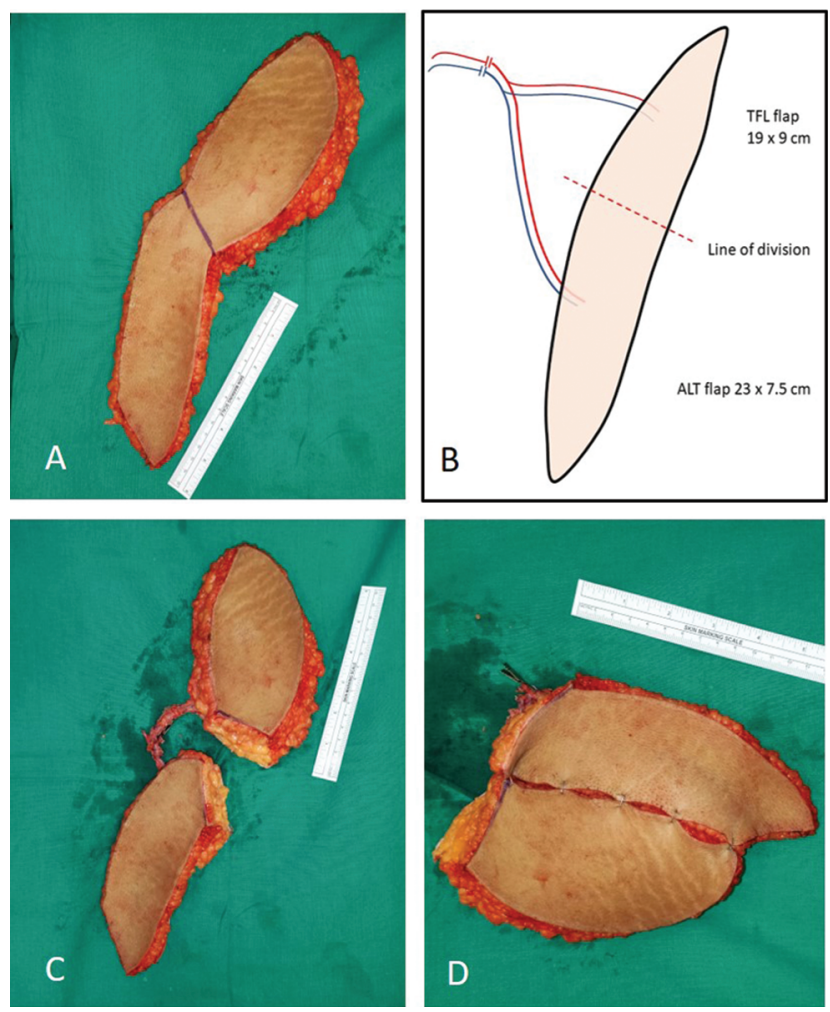

Fig. 3 (a) Harvested of anterolateral thigh (ALT) plus tensor fascia lata (TFL) flap. (b) Schematic representation of ALT plus TFL flap with both perforators joining to form a common pedicle at lateral circumflex femoral artery (LCFA), and planning of division. (c) Flap divided based on robust individual perforators. (d) Rotated and realigned ALT flap and TFL flap.

and axillary dissection. Resultant skin defect was $\sim 20 \mathrm{~cm} \times$ $18 \mathrm{~cm}$ ( - Fig. 2a). She had a pear-shaped body habitus and flaps from trunk donor sites would be grossly inadequate. The thigh had adequate girth for a single-island ALT perforator flap, but breast volume and shape would not be achieved and donor site would need large skin grafts. A decision to include proximal lateral thigh, which was much thicker and yielding more skin on primary closure, was taken based on TFL perforator. A chimeric ALT + TFL perforator flap if the pedicle were joining without any intertwining nerves, or two separate ALT + TFL free flaps otherwise, was planned. (-Fig. 2b).

\section{Flap Execution}

Planning in reverse was done using the template of skin defect. The template was divided into two, representing the ALT and TFL skin islands. The islands would contribute in such a way that each defect closes primarily. Hand-held audio Doppler $8 \mathrm{MHz}$ was used to scan for perforators.

An anterior, subfascial, noncommittal incision for ALT flap was planned $2 \mathrm{~cm}$ anterior to Doppler signal. A 2 to $3 \mathrm{~mm}-$ sized robust ALT perforator was found entering deep fascia and was dissected to its pedicle, descending branch of lateral circumflex femoral artery (LCFA). The anterior incision was extended cranially and posteriorly as the anterior incision for TFL flap. The search for TFL perforator was made in the plane between TFL muscle and overlying lateral deep fascial layer. The medial deep fascial layer was left in situ. The consistent robust $2 \mathrm{~mm}$ perforator emerging in the septum between TFL and gluteus medius muscle was found and traced to its pedicle, transverse branch of LCFA. The descending and transverse branches of LCFA and its vein were joining each other, yielding a $2 \mathrm{~cm}$ stump before meeting deep femoral vessels.

Posterior incision was committed after completion of perforator and pedicle dissection with flap dimensions of ALT $23 \mathrm{~cm} \times 7.5 \mathrm{~cm}$ and TFL $19 \mathrm{~cm} \times 9 \mathrm{~cm}$. Donor site was closed with minimal tension. The thickness of TFL flap was around thrice of ALT and would be mainly contributing to the bulk.

Flap was divided to make a chimeric flap ( - Fig. 3 ). The skin islands were rotated and realigned so that the TFL flap was in the lower part and the ALT flap in the upper part of the defect ( - Fig. 4). The second left internal mammary artery (IMA) and internal mammary vein (IMV) perforator were used as donor vessels.

\section{Follow-up}

All wounds healed uneventfully. The patient received $40 \mathrm{~Gy}$ of the planned $50 \mathrm{~Gy}$ of radiation, defaulting due to logistics compulsions out of the COVID-19 pandemic. At 10 months of follow-up, no debilitating effects of radiation were seen on skin. Scar stretching was seen on flap and thigh scars
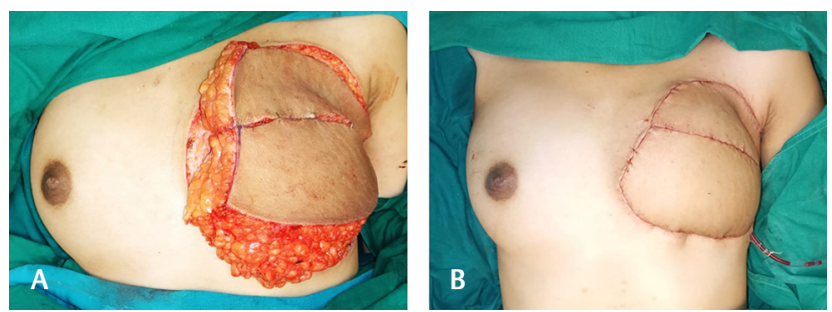

Fig. 4 (a,b) Thicker tensor fascia lata (TFL) flap formed the lower pole and relatively thinner anterolateral thigh (ALT) flap formed the upper pole. 

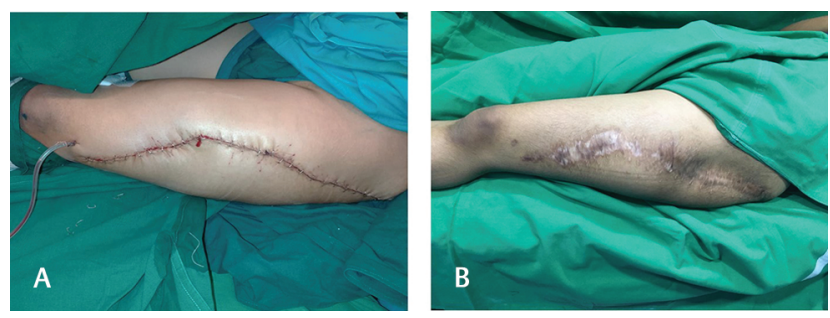

Fig. 5 Primary closure of the donor area without significant tension. Donor area after 6 months with scar stretching.
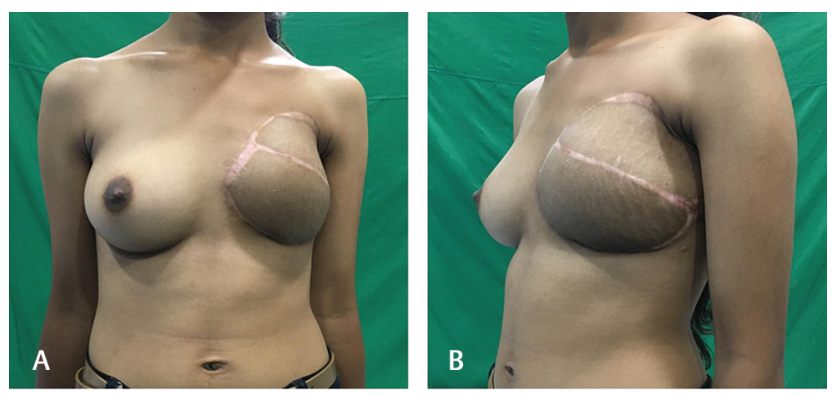

Fig. 6 Six months postoperative follow-up pictures.

( - Fig. 5). Volume of the reconstructed breast was nearly symmetrical to the contralateral side ( - Fig. $\mathbf{6}$ ).

\section{Discussion}

Large skin defects are often seen after advanced malignant phyllodes tumor excision. LD myocutaneous flap covers the skin defect often without a symmetrical mound. This might result in a large donor defect, requiring skin grafts. A laterally placed defect might overlap with donor site. ${ }^{2}$ Pedicle TRAM or freeDIEP/Transverse rectus abdominis myocutaneous (TRAM) flap can be used for the same, donor site permitting.

An ALT flap, described by Song, ${ }^{3}$ is the workhorse flap in reconstructive surgeries. A single large ALT flap can cover a large chest wall defect but has a few disadvantages. Perforator(s) may not robust enough to sustain the entire large flap, leading to partial necrosis. Closure of the donor site requires large skin grafts. It has limited ability to contour into a breast mound.

An anteromedial thigh (AMT) flap, described by Song et $\mathrm{al},{ }^{3}$ complements and completes an ALT flap. ${ }^{4}$ When used as chimeric flap with an ALT flap, extra skin and volume can be recruited but donor site requires skin grafts.

A TFL perforator flap described by Deiler $^{5}$ is an alternative to an ALT flap when suitable perforators were not found. ${ }^{6}$ Transverse branch of the LCFA gives very consistent and sizeable perforators in septum between the TFL and gluteus medius. ${ }^{7}$ The TFL perforator flap can be the harvested by same surgical access used for the ALT flap. ${ }^{2}$ TFL perforator flaps or lateral thigh flaps for breast reconstruction are well described as opportunistic alternative to traditional donor sites. ${ }^{8,9}$ The use of combined/split TFL and ALT flap together for cover of large chest wall defects following advanced breast malignancies has been described by Pelzer. ${ }^{10}$
In the described case, proximal TFL territory was significantly thicker than ALT. Inset of the TFL flap inferiorly was done to achieve natural fullness of lower breast pole. Thinner ALT flap is used for thinner upper pole. This placement of flaps gave more natural breast contour. Inclusion of separate perforators for each flap resulted in improved overall vascularity of flap, reducing chances of partial necrosis and fat necrosis.

\section{Conclusion}

The combined ALT + TFL perforator flaps as a chimeric flap or double free flap can be used for closure of large skin defects following mastectomy for advanced or recurrent disease, when conventional donor sites fall insufficient. The concept can be extended to reconstruct breast, achieving aesthetic goals too. Donor-site morbidity is reduced because of primary closure of donor areas.

\section{Financial disclosures}

None.

\section{Conflict of Interest}

None declared.

\section{References}

1 World Health Oragnization. Cancer country profiles 2020-India. https://www.who.int/cancer/country-profiles/IND_2020.pdf?ua=1. Accessed June 1, 2020

2 Billington A, Dayicioglu D, Smith P, Kiluk J. Review of procedures for reconstruction of soft tissue chest wall defects following advanced breast malignancies. Cancer Contr 2019;26(1):1073274819827284

3 Song YG, Chen GZ, Song YL. The free thigh flap: a new free flap concept based on the septocutaneous artery. Br J Plast Surg 1984;37(2):149-159

4 Jaiswal D, Ghalme A, Yadav P, Shankhdhar V, Deshpande A. Free anteromedial thigh perforator flap: complementing and completing the anterolateral thigh flap. Indian J Plast Surg 2017;50(1):16-20

5 Deiler S, Pfadenhauer A, Widmann J, Stutzle H, Kanz K-G, Stock W. Tensor fasciae latae perforator flap for reconstruction of composite Achilles tendon defects with skin and vascularized fascia. Plast Reconstr Surg 2000;106(2):342-349

6 Contedini F, Negosanti L, Pinto V, et al. Tensor fascia latae perforator flap: an alternative reconstructive choice for anterolateral thigh flap when no sizable skin perforator is available. Indian J Plast Surg 2013;46(1):55-58

7 Hubmer MG, Schwaiger N, Windisch G, et al. The vascular anatomy of the tensor fasciae latae perforator flap. Plast Reconstr Surg 2009;124(1):181-189

8 Tuinder SMH, Beugels J, Lataster A, et al. The lateral thigh perforator flap for autologous breast reconstruction: a prospective analysis of 138 flaps. Plast Reconstr Surg 2018;141(2):257-268

9 Gore SM, Akhavani MA, Kang N, Chana JS. Chest wall reconstruction using a turbocharged chimaeric anterolateral thigh flap. J Plast Reconstr Aesthet Surg 2008;61(4):438-441

10 Pelzer M, Germann G, Czermak C, Reichenberger M. Combined split ALT/TFL flap for soft tissue coverage in large thoracical defects [in German]. Der Chirurg 2014;85(1):42-45 